\title{
Schemi attuali di terapia con calcitriolo
}

\author{
C. Zoccali
}

\section{Divisione di Nefrologia e CNR, Centro di Fisiologia Clinica, Reggio Calabria}

a 1,25D3 e il PTH hanno stretti rapporti di reciproca influenza (Fig. 1). Il PTH stimola la produzione di 1,25D3 e questa a sua volta sopprime il PTH per due vie: una indiretta che passa attraverso un aumento dell'assorbimento intestinale di calcio e della calcemia, e una diretta tramite recettori specifici sulle ghiandole paratiroidi. Dei due potenziali meccanismi di soppressione del PTH, solo il primo è messo a frutto con la terapia orale. In altri termini, il calcitriolo orale fa diminuire i livelli circolanti di PTH soprattutto perché induce un aumento della calcemia. Nell'ultimo decennio gli sforzi si sono concentrati sul secondo meccanismo. Si è cercato cioè di ottimizzare i risultati della terapia attivando la soppressione diretta delle paratiroidi. I risultati molto favorevoli riportati con la somministrazione endovena da Slatopolsky nel 1984 (1) hanno costituito uno stimolo fondamentale per una valutazione più puntuale del potenziale terapeutico dell'1,25 vitD3. A distanza di 10 anni dalla segnalazione di Slatopolsky è tuttavia ancora controverso se la via endovena offre reali vantaggi rispetto alla via orale. In questa review metterò a confronto a) la farmacocinetica e la farmacodinamica delle due vie di somministrazione, orale ed endovena; b) le modalità di somministrazione [bolo (orale o endovena) e terapia orale continua]; c) l'efficacia terapeutica dei boli endovena e dei boli orali; d) la compliance e $\mathrm{i}$ costi.

\section{Farmacocinetica e Farmacodinamica}

Quando viene somministrata una dose orale di 1,25 vitD3 (Fig 2A) una certa

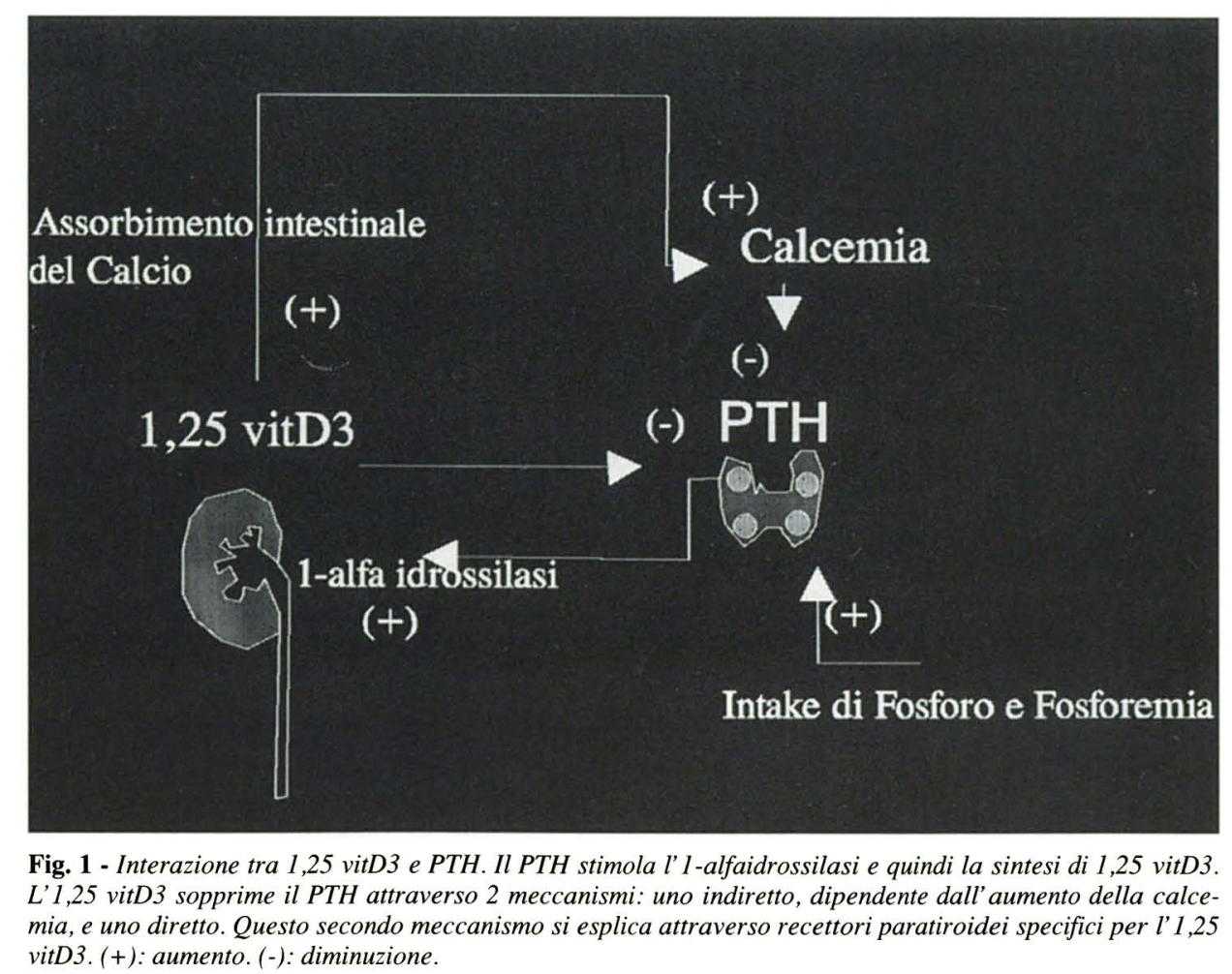

Fig. 1 - Interazione tra 1,25 vitD3 e PTH. Il PTH stimola l' l-alfaidrossilasi e quindi la sintesi di 1,25 vitD3. L'1,25 vitD3 sopprime il PTH attraverso 2 meccanismi: uno indiretto, dipendente dall aumento della calcemia, e uno diretto. Questo secondo meccanismo si esplica attraverso recettori paratiroidei specifici per $l$ ' 1,25 vitD3. (+): aumento. (-): diminuzione.

parte della dose viene immediatamente degradata a livello intestinale. Nel fegato, che è l'organo nel quale principalmente avviene il catabolismo dell'ormone, la vitamina arriva comunque in alta concentrazione attraverso il sangue portale. Il flusso ematico portale è poco più di 1 L e costituisce ben il $20 \%$ della portata cardiaca. Gran parte della dose viene 
2A

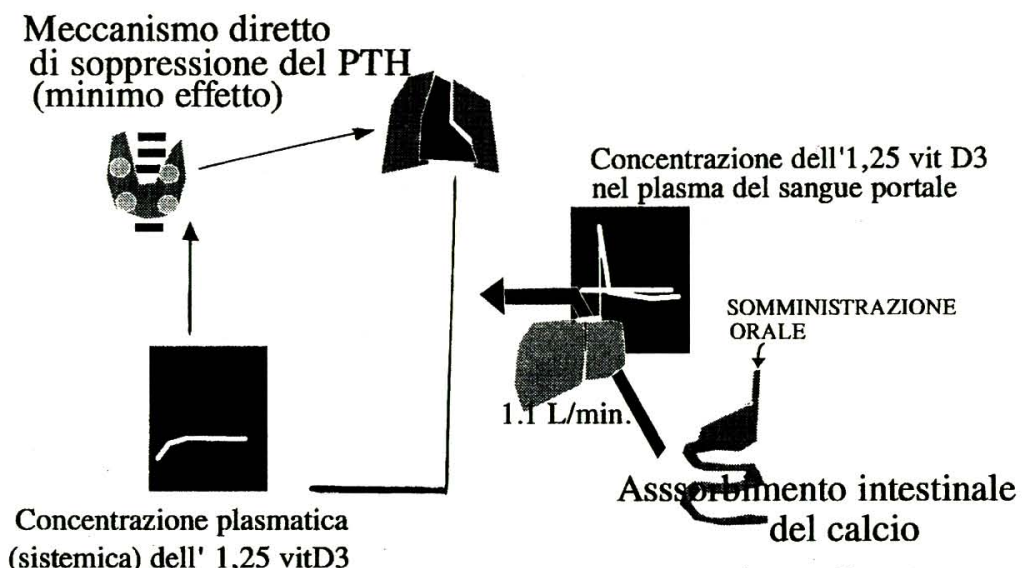

(massimo effetto)

2B

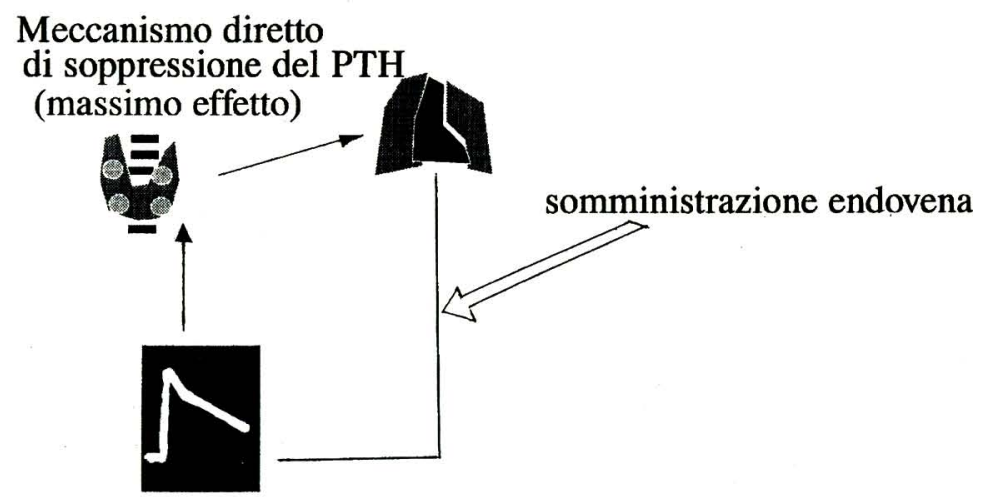

Concentrazione plasmatica

(sistemica) dell' 1,25 vitD3

Fig. 2 - Farmacocinetica delle due vie di somministrazione.

2A: La somministrazione orale determina uno spiccato aumento della concentrazione di 1,25 vitD3 nel sangue portale (grafico in nero, a destra). Tuttavia attraverso il transito epatico c'è un' alta degradazione metabolica di 1,25 vitD3 per cui la concentrazione plasmatica di questa sostanza aumenta solo di poco (grafico a sfondo nero, a sinistra). Ne deriva pertanto uno scarso effetto di soppressione diretta del PTH.

$2 B$ : La somministrazione endovena determina uno spiccato aumento della concentrazione plasmatica di 1,25 vitD3 e massimizza il meccanismo diretto di soppressione della secrezione paratiroidea.

pertanto degradata al "first pass" per cui alla circolazione generale perviene solo una frazione relativamente modesta della quantità somministrata. È importante tuttavia notare che l'intestino è direttamente esposto ad un'alta concentrazione di 1,25 vitD3 che stimola in maniera massimale la sintesi della proteina di trasporto del calcio così esaltando l'assorbimento intestinale del calcio. L'1,25D3 orale, pertanto, ha un effetto massimale sull'assorbimento del calcio ma ha limitate possibilità di sopprimere il PTH per via diretta in quanto raggiunge livelli ematici relativamente bassi.

Quando la stessa dose viene sommini- strata endovena (Fig. 2B) non c'è alcuna degradazione "first pass", e la vitamina raggiunge livelli ematici molto elevati, fino $\mathrm{a} 20$ volte più elevati del valore medio normale. Gli alti livelli ematici raggiunti con la via endovenosa privilegiano pertanto l'azione di soppressione diretta del PTH piuttosto che quella indiretta (mediata dall'aumento della calcemia). Va peraltro sottolineato che livelli ematici elevati possono essere raggiunti anche somministrando il farmaco per via orale a dosi molto elevate. In altri termini, se somministriamo dosi orali da 2 a 4 volte più alte rispetto a quelle somministrate endovena (cioè boli per via orale di $4-5$ $\mu \mathrm{g} / \mathrm{die}$ ), nonostante l'alta degradazione "first pass", si ottengono elevate concentrazioni plasmatiche del farmaco (comunque nettamente più basse rispetto a quelle raggiunte col bolo endovena). Anche con i boli per via orale, pertanto, può essere attivata la soppressione diretta del PTH, cioè quella indipendente dall'effetto ipercalcemico.

Analizziamo ora i tempi di risposta del $P T H$ a un bolo endovena di $1,25 v i t D 3$. Come risulta chiaro dalla Figura 3 non c'è alcuna variazione del PTH e della calcemia nelle 6 ore successive al bolo (2). Il bolo, quindi, non ha effetti rapidi sul PTH. L'effetto dell' 1,25 vitD3 sul PTH comincia ad essere evidente $24 \mathrm{~h}$ dopo e, quel che è più importante, si mantiene nel tempo.

Nella Figura 4 è riassunta la nostra esperienza in 14 pazienti, resistenti alla terapia orale, trattati con i boli per via venosa per periodi compresi tra 3 mesi e 1 anno. In un arco temporale compreso tra 1 e 3 mesi il PTH ha subito in media una riduzione del $20 \%$ e in ben 5 casi è rientrato nel range normale. La calcemia in media non era aumentata, tuttavia in 8 pazienti è stato necessario ridurre la concentrazione del calcio nel bagno di dialisi in quanto nell'insieme si sono verificati 30 episodi di ipercalcemia.

Un quesito importante è perché per sopprimere la sintesi del PTH negli uremici emodializzati debbano essere necessari livelli plasmatici di 1,25 vitD3 così elevati, livelli cioè farmacologici piuttosto che fisiologici. Ciò dipende dal fatto che nell'insufficienza renale cronica il numero dei recettori per l'1,25vitD3 si riduce nettamente. Il numero di questi recettori dipende infatti dai livelli circolanti dell' 1,25 vitD3. In altri termini, quando i livelli circolanti di 1,25 vitD3 sono ele- 


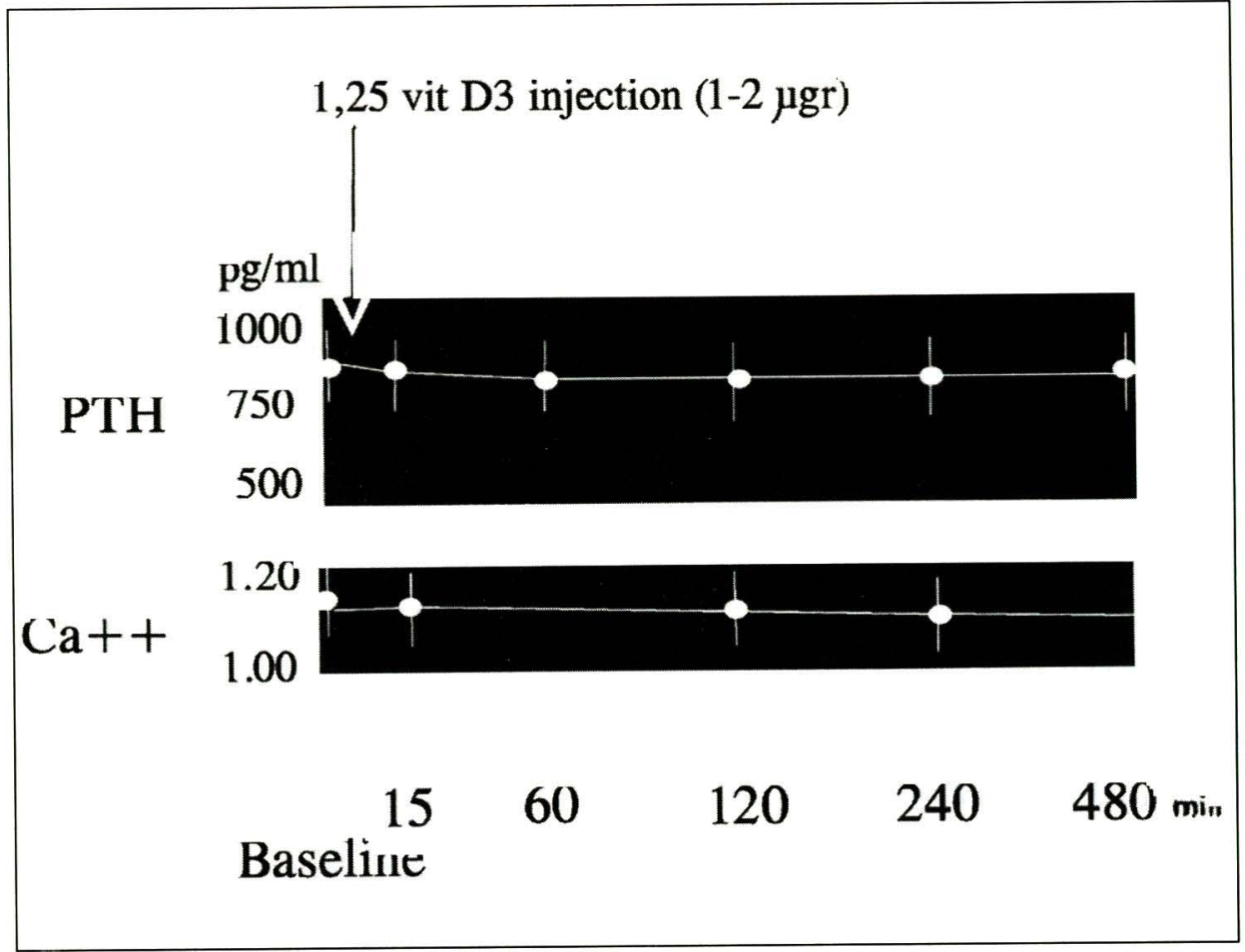

Fig. 3 - Assenza di effetti immediati (fino a 6 ore) di un bolo di 1,25 vitD3 sul PTH e sul calcio ionizzato. La linea tratteggiata delimita il range normale.

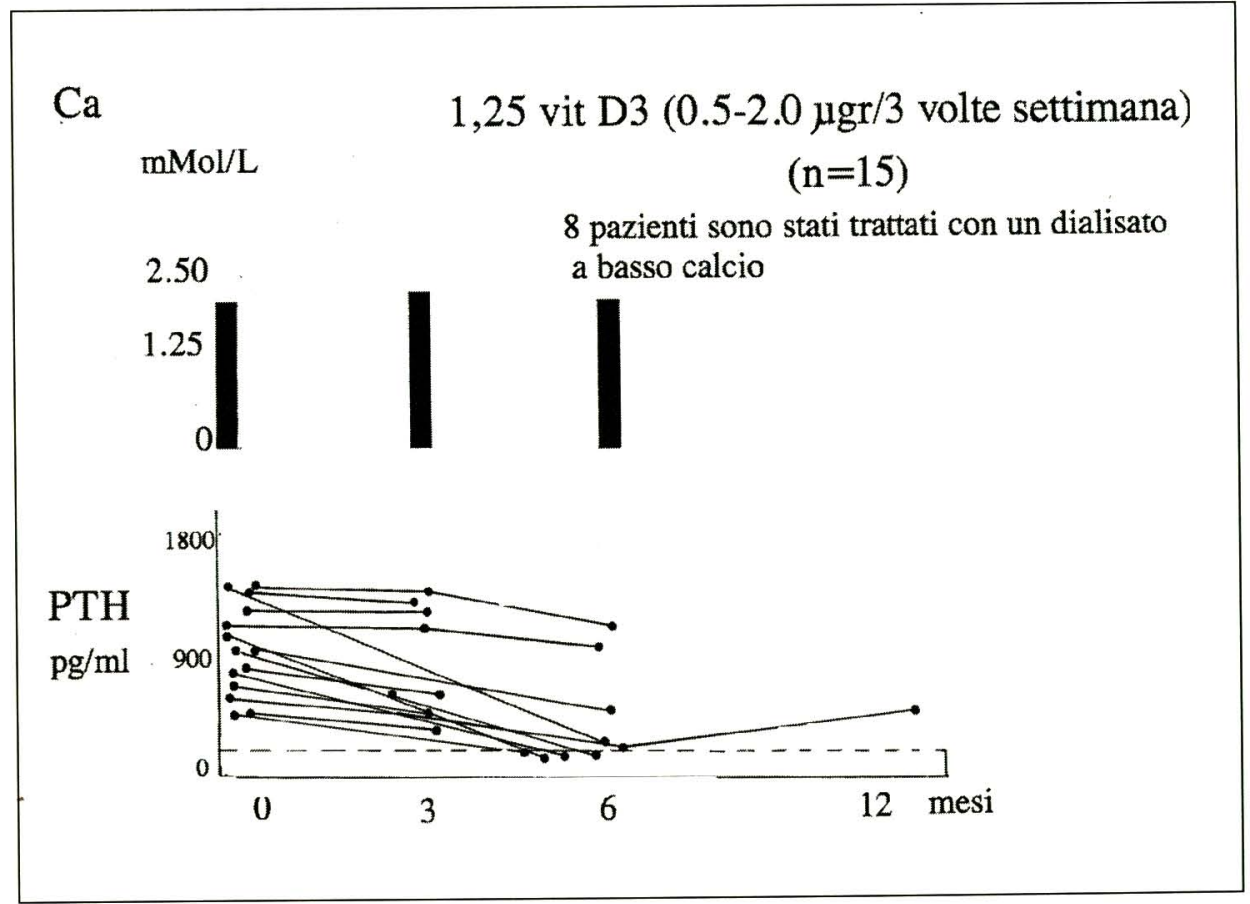

Fig. 4 - Effetti a lungo termine della terapia endovena in 15 pazienti. Le colonne del grafico superiore rappresentano la Calcemia media. vati aumenta il numero di recettori per 1,25 vitD3 e le paratiroidi diventano più sensibili al suo effetto soppressivo. Viceversa, quando i livelli circolanti sono bassi, i recettori diminuiscono di numero e la ghiandola diviene resistente all'1,25 vitD3.

\section{Efficacia relativa della terapia orale quotidiana e dei boli}

Alcuni studi indicano che $\mathrm{i}$ boli e.v. sono più efficaci della terapia orale. Andresse et al (3), in un gruppo di pazienti resistenti alla terapia orale, con l'uso dei boli e.v. hanno registrato una sostanziale diminuzione del turnover osseo, una diminuzione della superficie coperta da osteoide e della fibrosi midollare, tutti indici di un chiaro miglioramento dell'iperparatiroidismo. Tuttavia una soppressione paratiroidea quasi ottimale è stata ottenuta anche in alcuni studi in cui è stato impiegato un bolo orale (4). In questi casi l'iperparatiroidismo era meno grave rispetto a quello che presentavano i pazienti inclusi nello studio di Andress (3). D'altra parte, uno studio recente di Malluche (5) in pazienti non selezionati, non ha documentato alcun vantaggio dei boli endovena rispetto ai boli per os né in termini di controllo dell'iperparatiroidismo né in termini di effetti collaterali (episodi di ipercalcemia).

Riassumendo, i boli per via venosa sono probabilmente più efficaci nei casi più avanzati ma non sembrano avere un reale vantaggio sui boli orali nei casi di media gravità o nelle forme di iperparatiroidismo iniziale.

\section{Compliance e costi}

La compliance terapeutica è inversamente proporzionale al numero di pillole prescritte. È vicina al $100 \%$ quando si prescrive solo 1 pillola al giorno e declina fino a meno del $40 \%$ quando il numero di pillole è superiore a 6 . Dobbiamo essere ben consapevoli che quando prescriviamo il calcitriolo orale questo farmaco spesso va ad allungare una lista di prescrizioni già lunga (chelanti del calcio, farmaci anti-ipertensivi, supplementi vitaminici ecc). Nel nostro centro, un con- 


\section{compliance al calcitriolo orale in 72 pazienti in emodialisi ospedaliera}

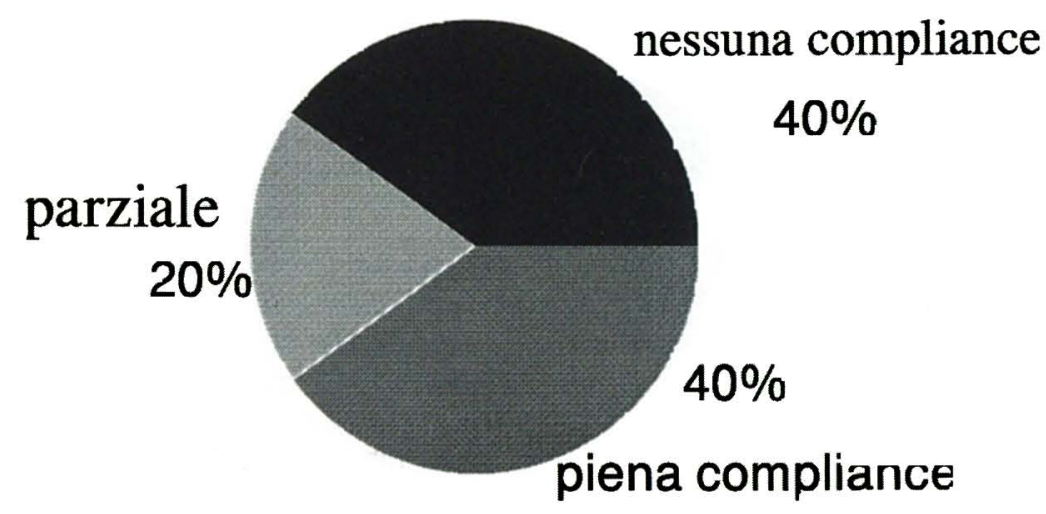

Fig. 5 - Compliance alla terapia orale con 1,25 vitD3 nella popolazione dialitica.

trollo random della compliance al calcitriolo ha mostrato che solo il $40 \%$ dei pazienti ha una compliance soddisfacente (Fig. 5). Un vantaggio dei boli endovena è che essi consentono di eliminare questo problema in quanto la somministrazione viene fatta appena dopo la seduta dialitica. In teoria, la somministrazione orale potrebbe essere anche altrettanto valida in quanto $\mathrm{i}$ pazienti potrebbero assumere un bolo orale al termine della dialisi sotto il diretto controllo degli infermieri. Tuttavia non bisogna sottovalutare il fatto che le attuali formulazioni del calcitriolo obbligano i pazienti ad assumere da 5 a 10 c. in unica soluzione. Fino a quando l'industria non produrrà una macrodose, il numero delle pillole è una reale limitazione all'uso dei boli orali.

Un elemento importante che il nefrologo deve prendere in considerazione quando decide circa la formulazione orale o quella endovena sono i costi. Infatti i boli endovena costano circa 30 volte più della terapia orale quotidiana $\mathrm{e}$ 12 volte più dei boli orali. In tempi critici di contenimento della spesa sanitaria i boli endovena vanno riservati ai casi nei quali né la terapia orale continua né

\section{BIBLIOGRAFIA}

1. Slatopolsky E, Weerts C, Thielan J, Horst R, Harter H, Martin KJ. Marked suppression of secondary hyperparathyroidism by intravenous administration of 1,25-dihydroxycholecalciferol in uremic patients. J Clin Invest 1984; 74: 213643.

2. Zoccali C, Parlongo S, Sicuso C, Messineo L, Finocchiaro P. The influence of 1,25 vit D3 on plasma ANF concentration in haemodialysis patients. Nephrol Dial Transplant 1994.

3. Andress DL, Norris KC, Coburn JW. Intravenous calcitriol in treatment of refractory osteitis fibrosa of chronic renal failure. N Engl J Med 1989; 321: 274-9.

4. Kwan JTC, Almond MK, Beer JC, Noonan K, Evans SJW, Cunnigham J. Pulse oral calcitriol in uremic patients: Rapid modification of parathyroid response to calcium. Nephrol Dial Transplant 1992; 7 : 829-34.

tuttavia, un impiego accurato dei boli endovena può evitare o differire la paratiroidectomia.
5. Faugerer MC, Friedler RM, Malluche HH. Efficacy and limitations of pulse i.v. and pulse oral 1,25 vit D3 therapy on bone disease in patients on chronic dialysis. J Am Soc Nephrol 1993; 4: 695. 